\title{
Generalized Rotne-Prager-Yamakawa approximation for Brownian dynamics in shear flow in bounded, unbounded, and periodic domains
}

\author{
Bogdan Cichocki, ${ }^{1, \text { a) }}$ Piotr Szymczak, ${ }^{1, \text { b) }}$ and Pawel J. Zuk, 2, c) \\ ${ }^{1)}$ Institute of Theoretical Physics, Faculty of Physics, University of Warsaw, Pasteura 5, 02-093 Warsaw, \\ Poland \\ 2) Department of Biosystems and Soft Matter, Institute of Fundamental and Technological Research, Polish Academy of Sciences, \\ Pawinskiego 5B, 02-106, Warsaw, Poland \\ 3) Department of Physics, Lancaster University, Lancaster LA1 4YB, United Kingdom
}

\begin{abstract}
Inclusion of hydrodynamic interactions is essential for a quantitatively accurate Brownian Dynamics simulation of colloidal suspensions or polymer solutions. We use the generalized Rotne-Prager-Yamakawa (GRPY) approximation, which takes into account all long-ranged terms in the hydrodynamic interactions, to derive the complete set of hydrodynamic matrices in different geometries: unbounded space, periodic boundary conditions of Lees-Edwards type and vicinity of a free surface. The construction is carried out both for non-overlapping as well as for overlapping particles. We include the dipolar degrees of freedom, which allows one to use this formalism to simulate the dynamics of suspensions in a shear flow and to study the evolution of their rheological properties. Finally, we provide an open-source numerical package which implements GRPY algorithm in Lees-Edwards periodic boundary conditions.
\end{abstract}

\section{INTRODUCTION}

Brownian dynamics is one of the most versatile and powerful methods of simulating biological and soft matter systems ${ }^{1-3}$. It treats the solvent implicitly, projecting out the fluid degrees of freedom and taking their effect into account through the correlations in the stochastic displacements. These are caused by the so-called hydrodynamic interactions (HI): the motion of one particle induces a flow which acts on all the other particles. As repeatedly demonstrated, the proper inclusion of hydrodynamic interactions is crucial for correct capturing of soft matter dynamics ${ }^{4-12}$. However, this is not an easy task due to the long ranged and multi-body character of HI. To make the problem tractable, one usually resorts to approximations. The minimum requirement for a good approximation is that it correctly represents all the long-range terms, decaying with a rate slower or equal to $r^{-d}$ at large distances, where $d$ is the spatial dimensionality of the system. For translational and rotational components, such an approximation was proposed by Rotne, Prager and Yamakawa ${ }^{13,14}$. The Rotne-Prager-Yamakawa (RPY) approximation performs well in far-field, but it becomes less accurate at smaller distances. In particular, when the particles overlap, the hydrodynamic tensors calculated based on RPY may become nonpositive definite, which causes a problem in the Brownian dynamics simulations where the square root of the mobility matrix is needed. Calculation of hydrodynamic tensors for overlapping particles is also important for bead models of rigid macromolecules ${ }^{15-19}$. Overlapping bead models allow for a faithful representation of complex macromolecular shapes using a significantly smaller number of beads than the nonoverlapping models.

Recently, we proposed a generalization of RPY approach $(\mathrm{GRPY})^{18,20,21}$ which works both for non-overlapping and

\footnotetext{
a)Electronic mail: cichocki@fuw.edu.pl

b)Electronic mail: piotr.szymczak@fuw.edu.pl

c)Electronic mail: pjzuk@ippt.pan.pl
}

overlapping particles, and guarantees that the resulting mobility matrix will be positive definite. However, as argued $\mathrm{in}^{22}$, when dipolar degrees of freedom are included, the correct derivation of GRPY should be based on the inverse friction matrix, $\boldsymbol{m}^{19,22}$ and not on the mobility matrix, $\boldsymbol{\mu}^{18,20}$. A similar, $\boldsymbol{m}$-based approach was taken by Durlofsky, Brady and Bossis in their construction of Stokesian Dynamics algorithm ${ }^{23,24}$

Both approaches lead to the same results if only translational and rotational components are considered but they differ in the problems involving dipolar degrees of freedom e.g. dynamics in shear flows and calculation of hydrodynamic stresses. Physically, these differences correspond to the fact that the particles placed in a shear flow modify the effective value of a shear rate. This is a very significant effect, which cannot be neglected even at relatively low volume fractions of suspended particles. Thus the range of applicability of $\boldsymbol{\mu}$ based approach is very limited and $\boldsymbol{m}$-based approach needs to be developed, which is the main goal of this study.

In this communication, we show how these ideas can be used for a construction of hydrodynamic matrices for Brownian dynamics simulation in the presence of a shear flow. We are building on our previous paper ${ }^{21}$, but with a revised scheme of calculating the dipolar elements of the hydrodynamic tensors, based on the inverse friction matrix. We derive a complete set of explicit formulae for the translational, rotational and dipolar components of the inverse friction matrix for nonoverlapping particles as well as regularizing corrections to these matrices accounting for particle overlaps. We also extend the method to the case of a general Green's functions of Stokes flow and carry out the construction for three different geometries of the system: unbounded space, free surface, and periodic boundary conditions of Lees-Edwards kind.

\section{HYDRODYNAMIC INTERACTIONS}

We consider a suspension of $N$ identical spherical particles of different radii $a_{i}$, in an incompressible fluid of viscosity $\eta$ 
at a low Reynolds number. The particles are immersed in an external flow $\boldsymbol{v}_{\infty}$.

Due to the linearity of the Stokes equations, translational and rotational velocities of the particles $\left(\boldsymbol{U}_{i}, \boldsymbol{\Omega}_{i}\right)$ depend linearly on the forces and torques exerted by the fluid on the particles $\left(\boldsymbol{F}_{j}\right.$ and $\left.\boldsymbol{T}_{j}\right)$. This relation defines the grand mobility matrix $\boldsymbol{\mu}$

$$
\left(\begin{array}{c}
\tilde{\boldsymbol{U}}-\tilde{\boldsymbol{v}}_{\infty} \\
\tilde{\boldsymbol{\Omega}}-\tilde{\boldsymbol{\omega}}_{\infty} \\
-\tilde{\boldsymbol{S}}
\end{array}\right)=\left(\begin{array}{ccc}
\boldsymbol{\mu}^{t t} & \boldsymbol{\mu}^{t r} & \boldsymbol{\mu}^{t d} \\
\boldsymbol{\mu}^{r t} & \boldsymbol{\mu}^{r r} & \boldsymbol{\mu}^{r d} \\
\boldsymbol{\mu}^{d t} & \boldsymbol{\mu}^{d r} & \boldsymbol{\mu}^{d d}
\end{array}\right) \cdot\left(\begin{array}{c}
\tilde{\boldsymbol{F}} \\
\tilde{\boldsymbol{T}} \\
\tilde{\boldsymbol{E}}_{\infty}
\end{array}\right) .
$$

The elements $\boldsymbol{\mu}^{p q}$ (with $p, q=t, r, d$ ) are the Cartesian tensors and the superscripts $t, r, d$ denote translational, rotational and dipolar components, respectively. Here, $\tilde{\boldsymbol{F}}=\left(\boldsymbol{F}_{1}, \boldsymbol{F}_{2}, \ldots, \boldsymbol{F}_{N}\right)$ are the $3 N$ dimensional vectors of forces with which particles act on the fluid and analogously for the torques $\tilde{T}$, as well as translational and rotational velocities of the particles, $\tilde{\boldsymbol{U}}=\left(\boldsymbol{U}_{1}, \ldots, \boldsymbol{U}_{N}\right)$ and $\tilde{\boldsymbol{\Omega}}=\left(\boldsymbol{\Omega}_{1}, \ldots, \boldsymbol{\Omega}_{N}\right)$. Next, $\tilde{\boldsymbol{v}}_{\infty}=$ $\left(\boldsymbol{v}_{\infty}\left(\boldsymbol{R}_{1}\right), \ldots, \boldsymbol{v}_{\infty}\left(\boldsymbol{R}_{N}\right)\right)$ are the values of external flow velocity calculated at the centers of the particles, $\boldsymbol{R}_{i}$. Similarly, $\tilde{\boldsymbol{\omega}}_{\infty}$ gives the vector of vorticities at the centers of the particles, with $\boldsymbol{\omega}_{\infty}=\frac{1}{2} \nabla \times \boldsymbol{v}_{\infty}$. Finally, $\tilde{\boldsymbol{E}}_{\infty}=\left(\boldsymbol{E}_{\infty}\left(\boldsymbol{R}_{1}\right), \ldots, \boldsymbol{E}_{\infty}\left(\boldsymbol{R}_{N}\right)\right)$ is the vector of strain rates, with $\boldsymbol{E}_{\infty}=\frac{1}{2}\left(\nabla \boldsymbol{v}_{\infty}+\left(\nabla \boldsymbol{v}_{\infty}\right)^{T}\right)$ and $T$ standing for transposition. Finally, $\tilde{\boldsymbol{S}}=\left(\boldsymbol{S}_{1}, \ldots, \boldsymbol{S}_{N}\right)$ are the particle stresses. Both $\tilde{\boldsymbol{E}}_{\infty}$ and $\tilde{\boldsymbol{S}}$ are $5 N$-dimensional, due to the symmetric and traceless character of strain and stress tensors.

Note that on the RHS of equation (1) we find two quantities related to the forces $(\tilde{\boldsymbol{F}}$ and $\tilde{\boldsymbol{T}})$ and one quantity related to the flow $\left(\tilde{\boldsymbol{E}}_{\infty}\right)$. Although seemingly inconsistent, such a choice reflects the quantities that can be controlled in most situations of practical interest (forces, torques and external flows acting on a particle). However, from the point of view of theoretical analysis, the inverse friction matrix, $\boldsymbol{m}$ plays a much more important role. This matrix, introduced by Durlofsky, Brady, and Bossis ${ }^{23}$ links force and velocity multipoles

$$
\left(\begin{array}{c}
\tilde{\boldsymbol{v}}_{\infty}-\tilde{\boldsymbol{U}} \\
\tilde{\boldsymbol{\omega}}_{\infty}-\tilde{\boldsymbol{\Omega}} \\
\tilde{\boldsymbol{E}}_{\infty}
\end{array}\right)=-\left(\begin{array}{lll}
\boldsymbol{m}^{t t} & \boldsymbol{m}^{t r} & \boldsymbol{m}^{t d} \\
\boldsymbol{m}^{r t} & \boldsymbol{m}^{r r} & \boldsymbol{m}^{r d} \\
\boldsymbol{m}^{d t} & \boldsymbol{m}^{d r} & \boldsymbol{m}^{d d}
\end{array}\right) \cdot\left(\begin{array}{c}
\tilde{\boldsymbol{F}} \\
\tilde{\boldsymbol{T}} \\
\tilde{\boldsymbol{S}}
\end{array}\right)
$$

The inverse friction matrix will play a central role in the construction of the generalized Rotne-Prager-Yamakawa approximation (GRPY), as detailed in the next section.

\section{BROWNIAN DYNAMICS IN A SHEAR FLOW}

We consider the particles immersed in an external linear shear flow

$$
\boldsymbol{v}_{\infty}(\boldsymbol{r})=\boldsymbol{K}_{\infty} \cdot \boldsymbol{r}
$$

where $\boldsymbol{K}_{\infty}$ is the constant velocity gradient matrix, e.g. for a simple shear flow

$$
\boldsymbol{K}_{\infty}=\left[\begin{array}{ccc}
0 & 0 & \dot{\gamma} \\
0 & 0 & 0 \\
0 & 0 & 0
\end{array}\right], \quad \dot{\gamma}=\text { const. }
$$

In such case the strain rate, $\boldsymbol{E}_{\infty}$, is simply a symmetric part of the $\boldsymbol{K}_{\infty}$ tensor.

The hydrodynamic tensors defined in Sec. II can then be used in the Brownian dynamics scheme describing the evolution of the position vector $\boldsymbol{R}_{i}$ and the direction vector $\hat{\boldsymbol{e}}_{i}$ (e.g. the magnetic dipole moment) of each particle $i=1, \ldots, N^{1}$

$$
\begin{aligned}
& \boldsymbol{R}_{i}(t+\Delta t)=\boldsymbol{R}_{i}(t)+\boldsymbol{K}_{\infty} \cdot \boldsymbol{R}_{i}(t) \Delta t \\
& +\left(\sum_{j} \boldsymbol{\mu}_{i j}^{t t} \cdot \boldsymbol{F}_{j}+\sum_{j} \boldsymbol{\mu}_{i j}^{t r} \cdot \boldsymbol{T}_{j}+\sum_{j} \boldsymbol{\mu}_{i j}^{t d}: \boldsymbol{E}_{\infty}\right) \Delta t+\boldsymbol{\Gamma}_{i}^{t},
\end{aligned}
$$

$$
\begin{aligned}
& \hat{\boldsymbol{e}}_{i}(t+\Delta t)=\hat{\boldsymbol{e}}_{i}(t)+\frac{1}{2} \boldsymbol{\epsilon}: \boldsymbol{K}_{\infty} \Delta t \times \hat{\boldsymbol{e}}_{i}(t) \\
& +\left[\sum_{j} \boldsymbol{\mu}_{i j}^{r t} \cdot \boldsymbol{F}_{j} \Delta t+\sum_{j} \boldsymbol{\mu}_{i j}^{r r} \cdot \boldsymbol{T}_{j} \Delta t+\sum_{j} \boldsymbol{\mu}_{i j}^{r d}: \boldsymbol{E}_{\infty} \Delta t+\boldsymbol{\Gamma}_{i}^{r}\right] \times \hat{\boldsymbol{e}}_{i}(t),
\end{aligned}
$$

where $\epsilon$ is the Levi-Civita tensor and : stands for tensor contraction. The stochastic displacement $\boldsymbol{\Gamma}$ is a Gaussian random variable with zero mean and the covariance

$$
\langle\boldsymbol{\Gamma}(\Delta t) \boldsymbol{\Gamma}(\Delta t)\rangle=2 \boldsymbol{D} \Delta t=2 k_{B} T \boldsymbol{\mu} \Delta t
$$

$$
k_{B} T \boldsymbol{\mu}=\mathcal{E} \cdot \mathcal{E}^{T}, \quad\left\langle g_{i} g_{j}\right\rangle=\delta_{i j}, \quad \boldsymbol{\Gamma}(\Delta t)=\sqrt{2 \Delta t} \mathcal{E} \cdot \boldsymbol{g}
$$

In the above we have neglected the terms involving divergence of mobility tensors ( $c f$. Ref. $\left.{ }^{1}\right)$, since they vanish within the RPY approximation. However, when one goes beyond RPY approximation and includes many-body effects in hydrodynamic interactions or introduces lubrication correction, the divergence of mobility matrix becomes non-zero and needs to be taken into account in Brownian dynamics simulation schemes ${ }^{25}$.

In general, there are two ways in which the uniform shear can be introduced in the molecular or Brownian dynamics simulations. The first is to confine the system between two parallel plates and translate one plate parallel to another at a constant speed. The disadvantage of such a setup is the presence of strong wall effects, which are undesirable, unless our intention is to model the flow in nanochannels. Alternatively, to mitigate wall effects and more effectively simulate the bulk, one uses the periodic cell of the Lees-Edwards kind $^{26,27}$, which deforms, becoming progressively more titled with time (cf. Fig. 1(a)). For a simple shear of a form $\dot{\gamma} z \mathbf{e}_{x}$ the tilting angle (i.e. the angle between the instantaneous direction of the tilted $z$ axis and its original direction) varies with the shear rate $\dot{\gamma}$ and time $t$ as ${ }^{27}$

$$
\theta=\arctan \left(\frac{\dot{\gamma} L_{z} t \bmod L_{x}}{L_{z}}\right)
$$

where $L_{x}, L_{y}$ and $L_{z}$ determine the initial size of the cell and mod stands for the modulo operation. Similarly, for the simulations elongational flow, Kraynik-Reinelt boundary conditions ${ }^{28}$ can be used. Again, the orthogonal cell is transformed into a parallelogram, albeit this time the deformation takes place along two orthogonal directions. 
(a)

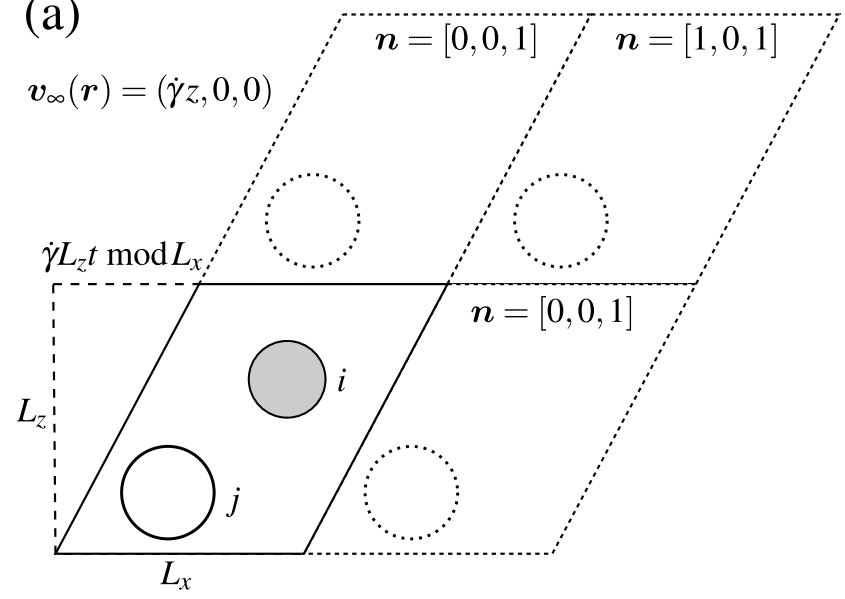

(b)

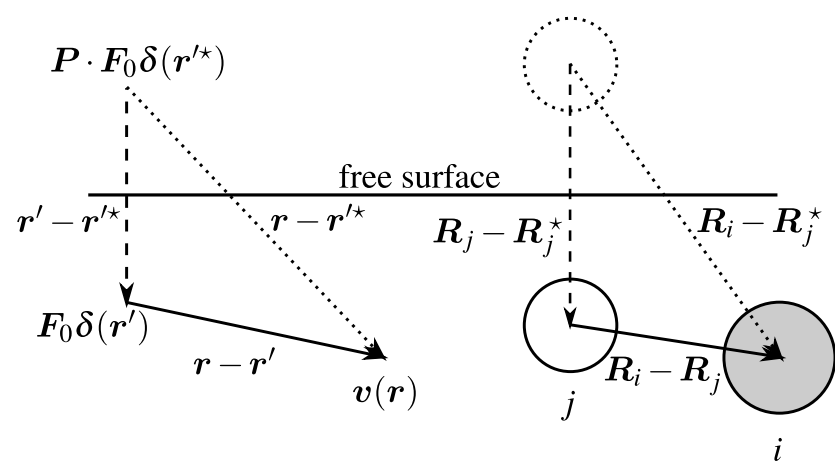

Newtonian fluid

FIG. 1. Schematics of different geometries of the system. (a) Lees-Edwards periodic boundary conditions for the shear flow. The tilt of periodic cells changes as a function of time. (b) Vicinity of a free surface. Grey particle ( $i$ ) feels the hydrodynamic disturbance generated by the white particle $j$ (solid line) as well as its images (dashed line).

\section{GENERALIZED ROTNE-PRAGER-YAMAKAWA APPROXIMATION}

The relation between the velocities of particles moving in a Stokes flow and induced force density localized on particle surfaces can be written as

$$
\left[\boldsymbol{U}_{i}+\boldsymbol{\Omega}_{i} \times \boldsymbol{\rho}_{i}-\boldsymbol{v}_{\infty}(\boldsymbol{r})\right]_{\boldsymbol{r} \in S_{i}}=\sum_{j} \int \boldsymbol{T}_{A}\left(\boldsymbol{r}-\boldsymbol{r}^{\prime}\right) \boldsymbol{f}_{j}\left(\boldsymbol{r}^{\prime}\right) \mathrm{d}^{3} \boldsymbol{r}^{\prime}
$$

Here $\boldsymbol{\rho}_{i}=\boldsymbol{r}-\boldsymbol{R}_{i}$ with $\boldsymbol{R}_{i}$ denoting the position of particle $i$. On the other hand, $-\boldsymbol{f}_{j}\left(\boldsymbol{r}^{\prime}\right)$ is the density of the forces with which the particle $j$ is acting on the fluid. Finally, $\boldsymbol{T}_{A}$ is the Green's function for the Stokes problem for a particular geometry. In the present study, we will consider specifically the case of unbounded space, where the Green's function is the Oseen tensor, $T_{O}$, periodic boundary conditions, where the fundamental solution is given by the Hasimoto tensor, $T_{H}$ and the vicinity of a free surface, with the tensor $T_{F}$ (see Sec. V for more details).

The key idea behind the GRPY approximation is to describe force density in terms of its three multipoles only:

$$
\boldsymbol{f}_{j}=\boldsymbol{w}_{j}^{t} \cdot \boldsymbol{F}_{j}+\boldsymbol{w}_{j}^{r} \cdot \boldsymbol{T}_{j}+\boldsymbol{w}_{j}^{d}: \boldsymbol{S}_{j}
$$

where $\boldsymbol{w}_{j}^{p}$ are operators associated with different multipoles $(p=t, r, d)$

$$
\begin{gathered}
\boldsymbol{w}_{j}^{t}(\boldsymbol{r})=\frac{1}{4 \pi a_{j}^{2}} \mathbf{1} \boldsymbol{\delta}\left(\rho_{j}-a_{j}\right), \quad \boldsymbol{w}_{j}^{r}(\boldsymbol{r})=\frac{3}{8 \pi a_{j}^{3}} \boldsymbol{\epsilon} \cdot \hat{\boldsymbol{\rho}}_{j} \boldsymbol{\delta}\left(\rho_{j}-a_{j}\right) \\
\boldsymbol{w}_{j}^{d}(\boldsymbol{r})=\frac{3}{4 \pi a_{j}^{3}} \boldsymbol{\delta}\left(\rho_{j}-a_{j}\right) \hat{\boldsymbol{\rho}}_{j} \cdot \mathcal{I} .
\end{gathered}
$$

In the above $\left[\boldsymbol{\epsilon} \cdot \hat{\boldsymbol{\rho}}_{j}\right]_{\alpha \beta}=\varepsilon_{\alpha \beta \gamma}\left[\hat{\boldsymbol{\rho}}_{j}\right]_{\gamma}$ and $\mathcal{I}$, is the fourth rank isotropic tensor, traceless and symmetric in its first and last index pairs:

$$
\mathcal{I}_{\alpha \beta \delta \gamma}=\frac{1}{2}\left(\delta_{\alpha \delta} \delta_{\beta \gamma}+\delta_{\alpha \gamma} \delta_{\beta \delta}-\frac{2}{3} \delta_{\alpha \beta} \delta_{\delta \gamma}\right)
$$

Next, the velocity field in the LHS of Eq. (8) is approximated by a linear flow. The multipoles characterizing this flow: velocity, $\boldsymbol{U}_{i}-\boldsymbol{v}_{\infty}\left(\boldsymbol{R}_{i}\right)$, vorticity, $\boldsymbol{\Omega}_{i}-\boldsymbol{\omega}_{\infty}$, and strain rate, $\boldsymbol{E}_{\infty}\left(\boldsymbol{R}_{i}\right)$, can be obtained from $\left[\boldsymbol{U}_{i}+\boldsymbol{\Omega}_{i} \times \boldsymbol{\rho}_{i}-\boldsymbol{v}_{\infty}(\boldsymbol{r})\right]_{\boldsymbol{r} \in S_{i}}$ by integration of this expression multiplied by the transposed $\boldsymbol{w}_{i}^{p}$ operators. This leads to the following relation for the elements of the inverse friction matrix

$$
\begin{aligned}
& \boldsymbol{m}_{A, i j}^{p q}=\left\langle\boldsymbol{w}_{i}^{p}\left|\boldsymbol{T}_{A}\right| \boldsymbol{w}_{j}^{q}\right\rangle \\
& \quad=\iint\left[\boldsymbol{w}_{i}^{p}\left(\boldsymbol{r}^{\prime}\right)\right]^{T} \cdot \boldsymbol{T}_{A}\left(\boldsymbol{r}^{\prime}-\boldsymbol{r}^{\prime \prime}\right) \cdot \boldsymbol{w}_{j}^{q}\left(\boldsymbol{r}^{\prime \prime}\right) \mathrm{d} \boldsymbol{r}^{\prime} \mathrm{d} \boldsymbol{r}^{\prime \prime}
\end{aligned}
$$

where we have used the relation (2) together with an approximation (9).

The inverse friction matrix is symmetric, thus

$$
\boldsymbol{m}_{i j}^{p q}=\left[\boldsymbol{m}_{j i}^{q p}\right]^{T}
$$

Finally, we need to keep in mind that the Brownian dynamics simulation uses the mobility matrix, $\boldsymbol{\mu}$, and not the inverse friction matrix, $\boldsymbol{m}$. The latter needs then to be partially inverted to get $\boldsymbol{\mu}$, which is the last step of the construction. This is a difference to the previous approach ${ }^{18,20,21}$ where the formulas analogous to (12) were used directly to generate the mobility matrix. As commented in the Introduction, if the problem does not entail dipolar degrees of freedom then both approaches lead to the same results. However, if the problem involves shear flow or calculation of particle stresses, then the $\boldsymbol{m}$-based approach is a correct one, since the particles placed in a shear flow modify the effective value of a shear rate. 


\section{FUNDAMENTAL SOLUTIONS}

\section{A. Unbounded space: Oseen tensor}

The Oseen tensor is a fundamental solution of the Stokes equations in unbounded domain

$$
\eta \nabla^{2} \boldsymbol{T}_{0}-\nabla \boldsymbol{Q}_{0}=-\mathbf{1} \boldsymbol{\delta}(\boldsymbol{r}), \quad \nabla \cdot \boldsymbol{T}_{0}=0
$$

and reads

$$
\boldsymbol{T}_{0}(\boldsymbol{r})=\frac{1}{8 \pi \eta_{0} r}(\mathbf{1}+\hat{\boldsymbol{r}} \hat{\boldsymbol{r}})
$$

The second tensor on the LHS in Eq. (14), $\boldsymbol{Q}_{0}$ gives the associated pressure:

$$
\boldsymbol{Q}_{0}(\boldsymbol{r})=\frac{1}{4 \pi r^{2}} \hat{\boldsymbol{r}}
$$

\section{B. Periodic case: Hasimoto tensor}

The generalization of (14) to the case of periodic boundary conditions is given by

$$
\eta \nabla^{2} \boldsymbol{T}_{H}-\nabla \boldsymbol{Q}_{H}=-\mathbf{1} \sum_{n_{1}, n_{2}, n_{3}}\left[\boldsymbol{\delta}\left(\boldsymbol{r}-\boldsymbol{r}_{n}\right)-1\right], \quad \nabla \cdot \boldsymbol{T}_{H}=0
$$

which defines the Hasimoto tensor, $\boldsymbol{T}_{H}(\mathbf{r})$ and its associated pressure tensor, $\boldsymbol{Q}_{H}(\mathbf{r})^{29}$. Here $\boldsymbol{n}=\left[n_{1}, n_{2}, n_{3}\right]$ is a vector of integers numbering the cells in the real lattice and $\boldsymbol{L} \cdot \boldsymbol{n}$ are the images of the origin in successive cells. In the above, $\boldsymbol{L}$ is the lattice matrix the columns of which are the lattice vectors.

Using Ewald summation technique, the Hasimoto tensor can be written as ${ }^{21,30-33}$

$$
\begin{aligned}
& \boldsymbol{T}_{\mathrm{H}}(\boldsymbol{r})=\sum_{\boldsymbol{n}}\left[\operatorname{erfc}\left(\frac{r_{\boldsymbol{n}}}{\sqrt{2 \sigma^{2}}}\right) \boldsymbol{T}_{0}\left(\boldsymbol{r}_{\boldsymbol{n}}\right)+\frac{\mathrm{e}^{-r_{\boldsymbol{n}}^{2} / 2 \sigma^{2}}}{4 \sqrt{2 \pi^{3}} \eta \sigma} \hat{\boldsymbol{r}}_{\boldsymbol{n}} \hat{\boldsymbol{r}}_{\boldsymbol{n}}\right]-\frac{\sigma^{2}}{2 \eta V} \mathbf{1}+\frac{1}{\eta V} \sum_{\boldsymbol{n}}^{\prime}\left[\mathbf{1}-\left(1+\frac{1}{2} \sigma^{2} k_{\boldsymbol{n}}^{2}\right) \hat{\boldsymbol{k}}_{\boldsymbol{n}} \hat{\boldsymbol{k}}_{\boldsymbol{n}}\right] \frac{\mathrm{e}^{-k_{\boldsymbol{n}}^{2} \sigma^{2} / 2}}{k_{\boldsymbol{n}}^{2}} \cos \left(\boldsymbol{k}_{\boldsymbol{n}} \cdot \boldsymbol{r}\right) . \\
& \boldsymbol{Q}_{\mathrm{H}}(\boldsymbol{r})=\sum_{n}\left[\operatorname{erfc}\left(\frac{r_{\boldsymbol{n}}}{\sqrt{2 \sigma^{2}}}\right) \boldsymbol{Q}_{0}\left(r_{\boldsymbol{n}}\right)+\frac{1}{r_{n}} \frac{\mathrm{e}^{-r_{n}^{2} / 2 \sigma^{2}}}{2 \sqrt{2 \pi^{3}} \sigma} \hat{\boldsymbol{r}}_{\boldsymbol{n}}\right]+\frac{1}{V} \sum_{\boldsymbol{n}}^{\prime} \hat{\boldsymbol{k}}_{\boldsymbol{n}} \frac{\mathrm{e}^{-k_{n}^{2} \sigma^{2} / 2}}{k_{n}} \sin \left(\boldsymbol{k}_{\boldsymbol{n}} \cdot \boldsymbol{r}\right)
\end{aligned}
$$

where $\sigma$ is a splitting parameter for the Ewald summations whereas

$$
r_{n}=r+L \cdot n
$$

and in the reciprocal lattice,

$$
\boldsymbol{k}_{\boldsymbol{n}}=2 \pi \boldsymbol{n} \cdot \boldsymbol{L}^{-1} .
$$

The prime at the summation symbol in (19) indicates that the term $\boldsymbol{n}=0$ is to be omitted.

In a simulation, the implementation of the Lees-Edwards boundary conditions requires that the lattice matrix depends on time, as given by Eq. (7). In the case at hand, for the coordinate axes defined as in (4), the lattice matrix takes the following form

$$
\boldsymbol{L}=\left[\begin{array}{ccc}
L_{x} & 0 & \dot{\gamma} L_{z} t \bmod L_{x} \\
0 & L_{y} & 0 \\
0 & 0 & L_{z}
\end{array}\right],
$$

We define the volume of basic cell as

$$
V=\operatorname{det} \boldsymbol{L}
$$

and the splitting parameter, $\sigma$ is taken to be

$$
\sigma=\frac{V^{1 / 3}}{\sqrt{2 \pi}}
$$

which corresponds to optimization of the numerical cost of real and reciprocal sum. Further details on performing the Ewald summation in skewed cells can be found in Ref. ${ }^{21}$.

\section{Free surface}

In the presence of boundaries, the Oseen solution gains an additional part describing the flow reflected from interfaces. For a free surface (cf. Fig. 1(b)), the flow field due to the force density localized at the source point $\boldsymbol{r}^{\prime}$, i.e. $\boldsymbol{f}(\boldsymbol{r})=\boldsymbol{F}_{0} \boldsymbol{\delta}(\boldsymbol{r}-$ $\left.\boldsymbol{r}^{\prime}\right)$, is given by ${ }^{34,35}$

$$
\begin{aligned}
\boldsymbol{v}(\boldsymbol{r})=\boldsymbol{T}_{\mathrm{F}}\left(\boldsymbol{r}, \boldsymbol{r}^{\prime}\right) \cdot \boldsymbol{F}_{0}=\boldsymbol{T}_{\mathrm{O}}( & \left.\boldsymbol{r}-\boldsymbol{r}^{\prime}\right) \cdot \boldsymbol{F}_{0} \\
& +\boldsymbol{T}_{\mathrm{O}}\left(\boldsymbol{r}-\boldsymbol{r}^{\prime \star}\right) \cdot \boldsymbol{P} \cdot \boldsymbol{F}_{0}
\end{aligned}
$$

where $\boldsymbol{P}=\mathbf{1}-2 \hat{\boldsymbol{e}}_{z} \hat{\boldsymbol{e}}_{z}$ denotes the reflection operator which transforms any point into its mirror image with respect to the surface and $\hat{e}_{z}$ a unit vector perpendicular to the surface. Finally, $\boldsymbol{r}^{\prime \star}=\boldsymbol{P} \cdot \boldsymbol{r}^{\prime}$ is the image of $\boldsymbol{r}^{\prime}$ with respect to the surface. 


\section{INVERSE FRICTION MATRIX}

The expression (12) allows one to calculate the inverse friction matrix for any propagator $\boldsymbol{T}$, corresponding to a particular geometry of the system. Below, we will consider specifically the cases discussed in Sec. V: unbounded space (Oseen tensor), the periodic system (Hasimoto tensor) or vicinity of a free surface. An analogous construction can be carried out for systems bounded by a cylinder and a sphere ${ }^{36-38}$, for the system bounded by two hard walls ${ }^{39}$ or elastic membrane ${ }^{40}$, in a liquid film between two fluids ${ }^{41,42}$, or any other system in which the fundamental solution can be derived.

The construction below is written out for a specific example of a Hasimoto tensor corresponding to periodic boundary conditions, but it can be applied to any of the tensors discussed above as long as it is characterized by the same singularities as the Oseen tensor as the particles begin to overlap.

Let us introduce the following auxiliary matrices

$$
\boldsymbol{M}_{A}^{p q}(\boldsymbol{r} ; a, b)=\boldsymbol{D}^{p}(\boldsymbol{r} ; a) \cdot \boldsymbol{T}_{A}(\boldsymbol{r}) \cdot \overleftarrow{\boldsymbol{D}^{q}}(\boldsymbol{r} ; b)
$$

where $p, q=t, r, d$.

The differential operators in (26) are given by

$$
\begin{gathered}
\boldsymbol{D}^{t}(\boldsymbol{r} ; a)=\left(1+\frac{a^{2}}{6} \nabla_{\boldsymbol{r}}^{2}\right) \boldsymbol{I}, \quad \boldsymbol{D}^{r}(\boldsymbol{r} ; a)=-\frac{1}{2} \varepsilon \cdot \nabla_{\boldsymbol{r}} \\
\boldsymbol{D}^{d}(\boldsymbol{r} ; a)=\left(1+\frac{a^{2}}{10} \nabla_{\boldsymbol{r}}^{2}\right) \mathcal{I}: \nabla_{\boldsymbol{r}}
\end{gathered}
$$

Next, the arrow above $\boldsymbol{D}$ means that the differential operator acts to the left, changing the sign of each differentiation, e.g. $f(r) \overleftarrow{\nabla}_{\boldsymbol{r}}=-\left(\boldsymbol{\nabla}_{\boldsymbol{r}} f(r)\right)$ and $f(r) \overleftarrow{\nabla}^{2}{ }_{\boldsymbol{r}}=\left(\boldsymbol{\nabla}^{2} \boldsymbol{r} f(r)\right)$

\section{A. Unbounded space}

In unbounded space, the self terms of the $\boldsymbol{m}$ matrix (corresponding to $i=j$ ) are simply single particle mobilities

$$
\boldsymbol{m}_{O, i i}^{p q}=\boldsymbol{m}_{0}^{p q}\left(a_{i}\right)
$$

Single particle mobilities, $m_{0}^{p q}$ are only nonzero for $p=q$ and read

$$
\begin{aligned}
\boldsymbol{m}_{0}^{t t}(a)=\frac{1}{6 \pi \eta a} \mathbf{1}, \quad \boldsymbol{m}_{0}^{r r}(a) & =\frac{1}{8 \pi \eta a^{3}} \mathbf{1} \\
\boldsymbol{m}_{0}^{d d}(a) & =\frac{3}{20 \pi \eta a^{3}} \mathcal{I} .
\end{aligned}
$$

For $i \neq j$ the form of the expression for the elements of the inverse friction matrix depends on the distance between the particles. For nonoverlapping particles, the elements of the inverse friction matrix, $m^{p q}$ are equal to the respective elements of the $M$ matrix:

$$
\boldsymbol{m}_{O, i j}^{p q}=\boldsymbol{M}_{O}^{p q}\left(\boldsymbol{R}_{i j} ; a_{i,} a_{j}\right) \quad R_{i j}>a_{i}+a_{j}
$$

$\boldsymbol{R}_{i j}=\boldsymbol{R}_{i}-\boldsymbol{R}_{j}$. On the other hand, for partially overlapping configurations ( $\left|a_{i}-a_{j}\right| \leq R_{i j} \leq a_{i}+a_{j}$ ), Eq. (26) can no longer be used and $\boldsymbol{m}^{p q}$ is calculated directly using the integral formula (12) $\left(\mathrm{see}^{22}\right)$

$$
\boldsymbol{m}_{O, i j}^{p q}=\boldsymbol{m}_{O, o v}^{p q}\left(\boldsymbol{R}_{i j} ; a_{i}, a_{j}\right)
$$

where we introduced the subscript $o v$ to emphasize that we use Eq. (12) for the overlapping particles. Finally, when the smaller particle becomes fully immersed in a larger one ( $\left.R_{i j} \leq\left|a_{i}-a_{j}\right|\right)$ then the shape of the surface over which we integrate (12) changes and the formulas for $\boldsymbol{m}^{p q}\left(R_{i j}\right)$ become much simpler. We will use subscript $i m$ to indicate such an immersed configuration.

Note that for $p=q$ the components $\boldsymbol{m}_{O, i m}^{p q}\left(a_{i}, a_{j}\right)$ of the inverse friction matrix can be directly obtained from the single particle mobilities, since

$$
\boldsymbol{m}_{O, i m}^{p q}\left(\boldsymbol{R}_{i j} ; a_{i}, a_{j}\right)=\boldsymbol{m}_{0}^{p q}\left(\max \left(a_{i}, a_{j}\right)\right), \quad p=q
$$

On the other hand, for $p \neq q$ the components $\boldsymbol{m}_{O, i m}^{p q}\left(a_{i}, a_{j}\right)$ read

$$
\begin{gathered}
\boldsymbol{m}_{O, i m}^{r t}(\boldsymbol{r} ; a, b)=\Theta(a-b) \boldsymbol{m}_{0}^{r r}(a) \cdot \boldsymbol{\epsilon} \cdot \boldsymbol{r} \\
\boldsymbol{m}_{O, i m}^{t d}(\boldsymbol{r} ; a, b)=\Theta(b-a) \boldsymbol{r} \cdot \boldsymbol{m}_{0}^{d d}(a)
\end{gathered}
$$

whereas $\boldsymbol{m}_{O, i m}^{r d}=0$.

\section{B. Periodic case}

Analogously to Eq. (30), for nonoverlapping particles in periodic boundary conditions (Fig. 1(a)) the inverse friction matrix can be expressed through the differential operators (26) as

$$
\boldsymbol{m}_{H, i j}^{p q}=\boldsymbol{M}_{H}^{p q}\left(\boldsymbol{R}_{i j} ; a_{i}, a_{j}\right) \quad R_{i j}>a_{i}+a_{j},
$$

The matrix elements of $\boldsymbol{M}_{H}^{p q}$ can be calculated using the Ewald summation formula for the Hasimoto tensor (19).

For partially overlapping particles $\left(\left(\left|a_{i}-a_{j}\right|<R_{i j} \leq a_{i}+\right.\right.$ $a_{j}$ ) we recover the elements of the inverse friction matrix by splitting the Hasimoto tensor as $\boldsymbol{T}_{H}=\boldsymbol{T}_{O}+\left(\boldsymbol{T}_{H}-\boldsymbol{T}_{O}\right)$ and calculating the respective contributions to the inverse friction matrix, using either the integral formula (12) (for the first term), or the differential formula (26) (for the second term):

$$
\boldsymbol{m}_{H, i j}^{p q}=\boldsymbol{m}_{O, o v}^{p q}\left(\boldsymbol{R}_{i j} ; a_{i}, a_{j}\right)+\boldsymbol{M}_{H O}^{p q}\left(\boldsymbol{R}_{i j} ; a_{i}, a_{j}\right)
$$

In the above, index $H O$ corresponds to putting into Eq. (26) the difference between the propagators

$$
\boldsymbol{T}_{H O}=\boldsymbol{T}_{H}-\boldsymbol{T}_{O}
$$

Note that $T_{H O}$ has no singularities at the particles overlap. The construction of $T_{H O}$ using the Ewald summation formula is described in the Appendix. 
The relation analogous to (36), although with $\boldsymbol{m}_{O, o v}$ replaced by $\boldsymbol{m}_{O, i m}$ is used to calculate $\boldsymbol{m}$ in the situation when one sphere is fully immersed within the other.

Finally, the self terms of the $\boldsymbol{m}$ matrix are:

$$
\boldsymbol{m}_{O, i i}^{p q}=\boldsymbol{m}_{0}^{p q}\left(a_{i}\right)+\boldsymbol{M}_{H O}^{p q}\left(\boldsymbol{R}_{i j}=0 ; a_{i}, a_{i}\right)
$$

Some of the components of the above-presented solution have been reported previously in the literature. In particular, the $t t$ component of the Rotne-Prager mobility matrix for periodic systems has been obtained by Beenakker $(1986)^{30}$ (see also Refs. ${ }^{31,32,43}$ ). Next, Jain et al. $(2012)^{44}$ have modified it to account for bead overlaps, but still for translational degrees of freedom only. On the other hand, Stoltz et al. $(2006)^{43}$ have considered the particles immersed in an external shear flow and the Lees-Edwards boundary conditions, however they again limited themselves to translational degrees of freedom only, neglecting the stresslet that arises as a particle is exposed to flow. The same regularizing correction for overlaps as derived above has also been used by Zhou \& Chen $(2006)^{45}$, but, again only in the case of translational degrees of freedom and in absence of external shear. Finally, as mentioned in the Introduction, Mizerski et al. ${ }^{21}$ have performed a similar construction to the one presented above, but based on the $\boldsymbol{\mu}$ matrix, which fails to take into account the modifications of the effective shear rate due to the presence of the particles. An approach closest to the present one, based on the $\boldsymbol{m}$ matrix was originally developed by Brady et al. (1988) as a part of the Stokesian Dynamics method ${ }^{32}$. Ref. ${ }^{32}$ considers equal-sized particles only and does not consider the regularizing corrections for particle overlaps. Subsequently, however, Fiore, Swan and collaborators ${ }^{46-48}$ have developed this approach further within so-called Fast Stokesian dynamics. Following the standard Ewald splitting into real and reciprocal space part, they subsequently approximate the real space sum by one term only, corresponding to the base cell, effectively assuming that the size of the cell is much larger than the inverse of the splitting parameter, $L \sigma \gg 1$. This allows them to calculate this part analytically. On the other hand, the reciprocal space part of the Ewald summation is calculated using fast Fourier transform technique, which requires a prior mapping of the particle force moment to a regular spatial grid. It is an alternative approach to the one presented here, well-suited to handle large numbers of particles in an efficient manner, but with a somewhat less controllable accuracy. We also note that in Refs. ${ }^{46-48}$ only translational and dipolar degrees of freedom are considered, and rotation is not included in the computational scheme.

\section{Free surface}

Since the fundamental solution in this case (Fig. 1(b)) can be expressed in a relatively simple way in terms of the Oseen tensor (Eq. 25), a similar decomposition holds for the elements of the inverse friction matrix ${ }^{49}$ (for $i \neq j$ )

$\boldsymbol{m}_{F, i j}^{p q}\left(\boldsymbol{R}_{i,} \boldsymbol{R}_{j}\right)=\boldsymbol{m}_{O, i j}^{p q}\left(\boldsymbol{R}_{i}-\boldsymbol{R}_{j}\right)+\boldsymbol{m}_{O, i j}^{p q}\left(\boldsymbol{R}_{i}-\boldsymbol{R}_{j}^{\star}\right) \cdot \boldsymbol{P}^{q}$ where $\boldsymbol{R}_{j}^{\star}$ is the image point defined analogously to that in Eq. (25) and

$$
\begin{aligned}
\boldsymbol{P}^{t}=\boldsymbol{P}, \quad \boldsymbol{P}^{r} & =-\boldsymbol{P} \\
P_{\alpha \beta \gamma \delta}^{d} & =\frac{1}{2}\left(P_{\alpha \gamma} P_{\beta \delta}+P_{\alpha \delta} P_{\beta \gamma}-\frac{2}{3} \delta_{\alpha \beta} \delta_{\gamma \delta}\right)
\end{aligned}
$$

The overlaps between the particles as well as between the particle and the wall can be accounted for by introducing the terms $\boldsymbol{m}_{O, o v}^{p q}$ whenever the arguments of the respective $\boldsymbol{m}_{O, i j}^{p q}$ function becomes smaller than $a_{i}+a_{j}$.

The self terms of the $\boldsymbol{m}$ matrix (corresponding to $i=j$ ) are calculated as

$$
\boldsymbol{m}_{F, i i}^{p q}\left(\boldsymbol{R}_{i}\right)=\boldsymbol{m}_{0}^{p q}\left(a_{i}\right)+\boldsymbol{m}_{O, i i^{*}}^{p q}\left(\boldsymbol{R}_{i}, a_{i}\right) \cdot \boldsymbol{P}^{q}
$$

where $z_{i}$ is the distance between the particle and the surface and $\boldsymbol{m}_{O, i i^{*}}^{p q}$ is the inverse friction matrix element for the particle $i$ and its image for unbounded space and is equal to

$$
\boldsymbol{m}_{O, i i^{*}}^{p q}= \begin{cases}\boldsymbol{M}_{O}^{p q}\left(\boldsymbol{R}_{i}-\boldsymbol{R}_{i}^{*} ; a_{i}, a_{i}\right), & z_{i}>a_{i} \\ \boldsymbol{m}_{O, o v}^{p q}\left(\boldsymbol{R}_{i}-\boldsymbol{R}_{i}^{*} ; a_{i}, a_{i}\right), & z_{i}<a_{i},\end{cases}
$$

where $z_{i}$ is the distance between the center of particle $i$ and the surface. Note that $\boldsymbol{R}_{i}-\boldsymbol{R}_{i}^{*}=2 z_{i} \hat{\boldsymbol{e}}_{z}$.

\section{CONCLUDING REMARKS}

We presented the reformulation of the GRPY approximation based on the inverse friction matrix and derived a complete set of all matrices $\boldsymbol{m}^{p q}$ for translational, rotational and dipolar degrees of freedom, including the regularizing corrections for overlapping particles. Latter can be used whenever due to the finite time step the particles overlap. In such a case standard formulas for mobility may lead to a matrix which is not positive definite leading to numerical problems in the Brownian dynamics simulations, where a square-root of the mobility matrix is needed. In the literature, such cases as dealt with in variety of ways: by introduction of a short-ranged repulsive potential to prevent the overlaps ${ }^{50-54}$, by rescaling the radii of the spheres for the sake of calculation $\boldsymbol{\mu}$ so that the gap between the particles becomes positive $e^{55-57}$ or to reflect the particles elastically each time they overlap ${ }^{25,57,58}$. The regularizing corrections derived above allow one to simply continue the simulations, without a need for extra rescaling or reflection. As noted in Refs. ${ }^{55-57}$ hydrodynamic interactions will cause the particle to leave the overlapping configuration in few time steps.

Additionally, the possibility of calculating hydrodynamic tensors for overlapping particles is important in the construc- 
tion of bead models of rigid macromolecules ${ }^{15-19}$. Such models allow for a faithful representation of complex macromolecular shapes using a significantly smaller number of beads than the nonoverlapping models.

Moreover, for the system with free surface we have shown how to take into account the overlaps of the particles with the surface. We did not give the formulas for hydrodynamic tensors in the presence of the hard wall, since these were provided by Swan and Brady ${ }^{59}$, but without the regularizing corrections for particle overlaps or for the overlap between particles and the wall. The former can be constructed relatively easily following the procedure outlined in Eq. (36). The derivation of the latter is needed for the Brownian Dynamics simulations in the vicinity of the wall ${ }^{60}$, but the proper account of overlaps is here significantly more complex than in the free surface case and will be the subject of the future work. We also note that a similar construction to ours (but for translational degrees of freedom only) has been carried out in Ref. ${ }^{42}$ for cylindrical objects embedded in a viscous sheet.

Finally, by inclusion of the dipolar degrees of freedom as well as Lees-Edwards boundary conditions the presented formalism can be used to study the rheological properties of complex fluids and non-equilibrium phenomena such as shearthickening or shear-thinning .

\section{DATA AVAILABILITY STATEMENT}

The Fortran implementation of GRPY algorithm in periodic boundary conditions is are available for download from github repository https://github.com/pjzuk/GRPerY. The examples included demonstrate the usage of this code both in Brownian and non-Brownian simulations.

\section{Appendix A: The elements of the inverse friction matrix for unbounded space and periodic boundary conditions}

In this Appendix, we give explicit expressions for $t, r$ and $d$ components of the inverse friction matrix with GRPY approximations and show construction of $T_{H O}$ tensor on the example of translational degrees of freedom. The formulas are given both for unbounded and periodic systems and for the cases of non-overlapping as well as overlapping particles. For the sake of brevity, we will only give the nonzero $m$ components. Additionally, we omit $d t, r t$ and $d r$ components, since they can be obtained from $t d, t r$ and $r d$ matrix elements using the symmetry of inverse friction matrix (Eq. (13)).

Components tt In the case of Oseen tensor, the auxilliary matrix (26) is as follows

$$
\boldsymbol{M}_{O}^{t t}(\boldsymbol{r} ; a, b)=\frac{1}{8 \pi \eta r}\left[\left(1+\frac{a^{2}+b^{2}}{3 r^{2}}\right) \mathbf{1}+\left(1-\frac{a^{2}+b^{2}}{r^{2}}\right) \hat{\boldsymbol{r}} \hat{\boldsymbol{r}}\right]
$$

According to Eq. (30) this also gives the elements of the inverse friction matrix, $\boldsymbol{m}_{O}^{t t}$ for nonoverlapping particles. For overlapping particles $(|a-b|<r \leq a+b)$ the inverse friction matrix is calculated according to Eq. (31) with

$$
\boldsymbol{m}_{O, o v}^{t t}(\boldsymbol{r} ; a, b)=\frac{1}{6 \pi \eta a b}\left[\frac{16 r^{3}(a+b)-\left((a-b)^{2}+3 r^{2}\right)^{2}}{32 r^{3}} \mathbf{1}+\frac{3\left((a-b)^{2}-r^{2}\right)^{2}}{32 r^{3}} \hat{\boldsymbol{r}} \hat{\boldsymbol{r}}\right] .
$$

Next, when the smaller particle is fully immersed in a larger one (for $r \leq|a-b|$ ) the the matrix element is no longer dependent on $\mathbf{r}$ and attains the form given by Eq. (32).

Next we move to the periodic (Hasimoto) case. Then

$$
\begin{aligned}
& \boldsymbol{M}_{H}^{t t}(\boldsymbol{r} ; a, b)=\sum_{\boldsymbol{n}}\left\{\boldsymbol{M}_{O}^{t t}\left(\boldsymbol{r}_{\boldsymbol{n}} ; a, b\right) \operatorname{erfc}\left(\frac{r_{\boldsymbol{n}}}{\sqrt{2 \sigma^{2}}}\right)+\left[\left(a^{2}+b^{2}\right)\left(\frac{1}{6} \sigma^{-2}+\frac{1}{3} r_{\boldsymbol{n}}^{-2}\right) \mathbf{1}+\left(\left(a^{2}+b^{2}\right)\left(\frac{1}{6} r_{\boldsymbol{n}}^{2} \sigma^{-4}-\frac{1}{3} \sigma^{-2}-r_{\boldsymbol{n}}^{-2}\right)+1\right) \hat{\boldsymbol{r}}_{\boldsymbol{n}} \hat{\boldsymbol{r}}_{\boldsymbol{n}}\right]\right. \\
& \left.\times \frac{1}{4 \sqrt{2 \pi^{3}} \eta \sigma} \mathrm{e}^{-r_{\boldsymbol{n}}^{2} / 2 \sigma^{2}}\right\}-\frac{\sigma^{2}}{2 \eta V} \mathbf{1}+\frac{1}{\eta V} \sum_{\boldsymbol{n}}^{\prime}\left[\mathbf{1}-\left(1+\frac{1}{2} \sigma^{2} k_{\boldsymbol{n}}^{2}\right) \hat{\boldsymbol{k}}_{\boldsymbol{n}} \hat{\boldsymbol{k}}_{\boldsymbol{n}}\right]\left(k_{\boldsymbol{n}}^{-2}-\frac{a^{2}+b^{2}}{6}\right) \mathrm{e}^{-k_{\boldsymbol{n}}^{2} \sigma^{2} / 2} \cos \left(\boldsymbol{k}_{\boldsymbol{n}} \cdot \boldsymbol{r}\right),
\end{aligned}
$$

For an overlapping configuration, we use the construction (36) to calculate the inverse friction matrix. The key element here is $\boldsymbol{M}_{H O}^{t t}$ which can be calculated by the formula analogous to (A2) but with the the first term in the sum (for $\mathbf{n}=0$ ) replaced by $\boldsymbol{M}_{O}^{t t}(\boldsymbol{r} ; a, b)\left(\operatorname{erfc}\left(\frac{r}{\sqrt{2 \sigma^{2}}}\right)-1\right)=-\boldsymbol{M}_{O}^{t t}(\boldsymbol{r} ; a, b) \operatorname{erf}\left(\frac{r}{\sqrt{2 \sigma^{2}}}\right)$, i.e.

$$
\boldsymbol{M}_{H O}^{t t}(\boldsymbol{r} ; a, b)=-\boldsymbol{M}_{O}^{t t}(\boldsymbol{r} ; a, b) \operatorname{erf}\left(\frac{r}{\sqrt{2 \sigma^{2}}}\right)+\sum_{\boldsymbol{n}}^{\prime} \boldsymbol{M}_{O}^{t t}\left(\boldsymbol{r}_{\boldsymbol{n}} ; a, b\right) \operatorname{erfc}\left(\frac{r_{\boldsymbol{n}}}{\sqrt{2 \sigma^{2}}}\right)+\sum_{\boldsymbol{n}}\left[\left(a^{2}+b^{2}\right)\left(\frac{1}{6} \sigma^{-2}+\frac{1}{3} r_{\boldsymbol{n}}^{-2}\right) \mathbf{1}+\ldots\right.
$$

Finally, the self terms are constructed using Eq. (38), i.e. putting $a=b$ and $r=0$ into Eq. (A3) and then adding the single particle mobility, $\boldsymbol{m}_{0}^{t t}$. This might seem problematic, because the individual terms corresponding to $\mathbf{n}=0$ in (A2) are diverging 
as $r \rightarrow 0$. However, together they converge to a finite limit, which we denote by $\widetilde{\boldsymbol{M}}_{H O}^{t t}(a)$. Explicitly

$$
\begin{array}{r}
\widetilde{\boldsymbol{M}}_{H O}^{t t}(a)=\lim _{\boldsymbol{r} \rightarrow 0}\left\{-\boldsymbol{M}_{O}^{t t}(\boldsymbol{r} ; a, a) \operatorname{erf}\left(\frac{r}{\sqrt{2 \sigma^{2}}}\right)+\frac{1}{4 \sqrt{2 \pi^{3}} \eta \sigma}\left[\frac{a^{2}}{3}\left(\frac{\sigma^{-2}}{2}+r^{-2}\right) \mathbf{1}+\left(2 a^{2}\left(-\frac{\sigma^{-2}}{3}-r^{-2}\right)+1\right) \hat{\boldsymbol{r}} \hat{\boldsymbol{r}}\right]\right\} \\
=\frac{1}{4 \sqrt{2 \pi^{3}} \sigma \eta}\left(-1+\frac{a^{2}}{9 \sigma^{2}}\right) \mathbf{1}
\end{array}
$$

The construction of other components of the inverse friction matrix is analogous to the one presented above for $t t$ components. For the sake of brevity, we give below only the final formulas for the other components.

$$
\begin{aligned}
& \boldsymbol{M}_{O}^{r r}(\boldsymbol{r} ; a, b)=\frac{1}{16 \pi \eta r^{3}}(3 \hat{\boldsymbol{r}} \hat{\boldsymbol{r}}-\mathbf{1}) \\
& \boldsymbol{m}_{O, o v}^{r r}(\boldsymbol{r} ; a, b)=\frac{1}{512 \pi \eta a^{3} b^{3} r^{3}}\left(s^{0}(r ; a, b) \mathbf{1}+s^{1}(r ; a, b) \hat{\boldsymbol{r}} \hat{\boldsymbol{r}}\right) \\
& s^{0}(r ; a, b)=5 r^{6}-27 r^{4}\left(a^{2}+b^{2}\right)+32 r^{3}\left(a^{3}+b^{3}\right)-9 r^{2}\left(a^{2}-b^{2}\right)^{2}-(a-b)^{4}\left(a^{2}+4 b a+b^{2}\right), \\
& s^{1}(r ; a, b)=3\left((a-b)^{2}-r^{2}\right)^{2}\left(a^{2}+4 b a+b^{2}-r^{2}\right) \text {. } \\
& \boldsymbol{M}_{H}^{r r}(\boldsymbol{r})=\sum_{\boldsymbol{n}}\left\{\boldsymbol{M}_{O}^{r r}\left(\boldsymbol{r}_{\boldsymbol{n}} ; a, b\right) \operatorname{erfc}\left(\frac{r_{\boldsymbol{n}}}{\sqrt{2 \sigma^{2}}}\right)-\left[\left(\frac{1}{4} \sigma^{-2}+\frac{1}{2} r_{n}^{-2}\right) \mathbf{1}+\left(\frac{1}{4} \sigma^{-4} r_{\boldsymbol{n}}^{2}-\frac{1}{2} \sigma^{-2}-\frac{3}{2} r_{\boldsymbol{n}}^{-2}\right) \hat{\boldsymbol{r}}_{\boldsymbol{n}} \hat{\boldsymbol{r}}_{\boldsymbol{n}}\right] \frac{1}{4 \sqrt{2 \pi^{3}} \eta \sigma} \mathrm{e}^{-r_{\boldsymbol{n}}^{2} / 2 \sigma^{2}}\right\} \\
& +\frac{1}{4 \eta V} \sum_{n}^{\prime}\left[1-\left(1+\frac{1}{2} \sigma^{2} k_{n}^{2}\right) \hat{\boldsymbol{k}}_{\boldsymbol{n}} \hat{\boldsymbol{k}}_{\boldsymbol{n}}\right] \mathrm{e}^{-k_{n}^{2} \sigma^{2} / 2} \cos \left(\boldsymbol{k}_{\boldsymbol{n}} \cdot \boldsymbol{r}\right) \\
& \widetilde{M}_{H O}^{r r}(a)=-\frac{1}{48{\sqrt{2 \pi^{3}}}^{3} \eta} \mathbf{1} \\
& \boldsymbol{M}_{O}^{r t}(\boldsymbol{r} ; a, b)=\frac{1}{8 \pi \eta r^{2}} \boldsymbol{\epsilon} \cdot \hat{\boldsymbol{r}} \\
& \boldsymbol{m}_{O, o v}^{r t}(\boldsymbol{r} ; a, b)=\frac{1}{128 \pi \eta a^{3} b r^{2}}(a-b+r)^{2}\left(b^{2}+2 b(a+r)-3(a-r)^{2}\right) \boldsymbol{\epsilon} \cdot \hat{\boldsymbol{r}} \\
& \boldsymbol{M}_{H}^{r t}(\boldsymbol{r} ; a, b)=\sum_{\boldsymbol{n}}\left[\boldsymbol{M}_{O}^{r t}\left(\boldsymbol{r}_{\boldsymbol{n}} ; a, b\right) \operatorname{erfc}\left(\frac{r_{\boldsymbol{n}}}{\sqrt{2 \sigma^{2}}}\right)+\frac{\boldsymbol{\epsilon} \cdot \hat{\boldsymbol{r}}_{\boldsymbol{n}}}{r_{\boldsymbol{n}}} \frac{1}{4 \sqrt{2 \pi^{3}} \eta \sigma} \mathrm{e}^{-r_{\boldsymbol{n}}^{2} / 2 \sigma^{2}}\right]+\frac{1}{2 \eta V} \sum_{\boldsymbol{n}}^{\prime} \frac{\boldsymbol{\epsilon} \cdot \hat{\boldsymbol{k}}_{\boldsymbol{n}}}{k_{\boldsymbol{n}}} \mathrm{e}^{-k_{\boldsymbol{n}}^{2} \sigma^{2} / 2} \sin \left(\boldsymbol{k}_{\boldsymbol{n}} \cdot \boldsymbol{r}\right) \\
& \boldsymbol{M}_{O}^{t d}(\boldsymbol{r}, a, b)=\frac{3}{20 \pi \eta b^{2}}\left[h^{0}(r ; a, b) \boldsymbol{p}^{0}(\hat{\boldsymbol{r}})+h^{1}(r ; a, b) \boldsymbol{p}^{1}(\hat{\boldsymbol{r}})\right] \\
& p_{\alpha \beta \gamma}^{0}(\hat{\boldsymbol{r}})=\delta_{\alpha \beta} \hat{r}_{\gamma}+\delta_{\alpha \gamma} \hat{r}_{\beta}-\frac{2}{3} \delta_{\beta \gamma} \hat{r}_{\alpha}, \quad p_{\alpha \beta \gamma}^{1}(\hat{\boldsymbol{r}})=\hat{r}_{\alpha}\left(\hat{r}_{\beta} \hat{r}_{\gamma}-\frac{1}{3} \delta_{\beta \gamma}\right) \\
& h_{O}^{0}(r ; a, b)=\frac{1}{6} b^{2}\left(5 a^{2}+3 b^{2}\right) r^{-4}, \quad h_{O}^{1}(r ; a, b)=\frac{5}{6} b^{2}\left(3 r^{-2}-\left(5 a^{2}+3 b^{2}\right) r^{-4}\right)
\end{aligned}
$$

The matrix $\boldsymbol{m}_{O, o v}^{t d}(\boldsymbol{r}, a, b)$ is given by a formula analogous to (A13) but with $h^{0}(r ; a, b)$ and $h^{1}(r ; a, b)$ replaced by

$$
\begin{aligned}
& h_{O, o v}^{0}(r ; a, b)=-\frac{1}{96 a b r^{4}}\left(10 r^{6}-24 a r^{5}-15 r^{4}(b-a)(b+a)+(b-a)^{5}(a+5 b)\right), \\
& h_{O, o v}^{1}(r ; a, b)=-\frac{5}{96 a b r^{4}}\left((a-b)^{2}-r^{2}\right)^{2}\left((a-b)(a+5 b)-r^{2}\right),
\end{aligned}
$$




$$
\begin{gathered}
\boldsymbol{M}_{H}^{t d}(\boldsymbol{r} ; a, b)=\sum_{\boldsymbol{n}}\left\{\boldsymbol{M}_{O}^{t d}\left(\boldsymbol{r}_{\boldsymbol{n}}, a, b\right) \operatorname{erfc}\left(\frac{r_{\boldsymbol{n}}}{\sqrt{2 \sigma^{2}}}\right)+\left[\chi^{0}\left(r_{\boldsymbol{n}}, a, b\right) \boldsymbol{p}^{0}\left(\hat{\boldsymbol{r}}_{\boldsymbol{n}}\right)+\chi^{1}\left(r_{\boldsymbol{n}}, a, b\right) \boldsymbol{p}^{1}\left(\hat{\boldsymbol{r}}_{\boldsymbol{n}}\right)\right] \frac{1}{4 \sqrt{2 \pi^{3}} \eta \sigma} \mathrm{e}^{-r_{\boldsymbol{n}}^{2} / 2 \sigma^{2}}\right\} \\
+\frac{1}{2 \eta V} \sum_{\boldsymbol{n}}^{\prime}\left[\boldsymbol{p}^{0}\left(\hat{\boldsymbol{k}}_{\boldsymbol{n}}\right)-\left(2+\sigma^{2} k_{\boldsymbol{n}}^{2}\right) \boldsymbol{p}^{1}\left(\hat{\boldsymbol{k}}_{\boldsymbol{n}}\right)\right]\left(1-\frac{5 a^{2}+3 b^{2}}{30} k_{\boldsymbol{n}}^{2}\right) \frac{1}{k_{\boldsymbol{n}}} \mathrm{e}^{-k_{\boldsymbol{n}}^{2} \sigma^{2} / 2} \sin \left(\boldsymbol{k}_{\boldsymbol{n}} \cdot \boldsymbol{r}\right) \\
\chi^{0}(r ; a, b)=\frac{1}{15}\left(5 a^{2}+3 b^{2}\right)\left(\sigma^{-2} r_{\boldsymbol{n}}^{-1}+3 r_{\boldsymbol{n}}^{-3}\right) \\
\chi^{1}(r ; a, b)=\left(5 a^{2}+3 b^{2}\right)\left(\frac{1}{30} r_{n}^{3} \sigma^{-6}-\frac{1}{15} \sigma^{-4} r_{\boldsymbol{n}}-\frac{1}{3} \sigma^{-2} r_{\boldsymbol{n}}^{-1}-r_{\boldsymbol{n}}^{-3}\right)+\sigma^{-2} r_{\boldsymbol{n}}+3 r_{\boldsymbol{n}}^{-1}
\end{gathered}
$$

$$
\begin{array}{r}
\boldsymbol{M}_{O}^{r d}(\boldsymbol{r} ; a, b)=\frac{3}{16 \pi \eta r^{3}} \boldsymbol{q}(\hat{\boldsymbol{r}})(\mathrm{A} 19) \quad \boldsymbol{m}_{O, o v}^{r d}(\boldsymbol{r} ; a, b)=\frac{3}{512 \pi \eta b^{3} a^{3} r^{3}}\left((a-b)^{2}-r^{2}\right)^{2}\left(a^{2}+4 b a+b^{2}-r^{2}\right) \boldsymbol{q}(\hat{\boldsymbol{r}}) \\
q_{\alpha \beta \gamma}(\hat{\boldsymbol{r}})=\hat{r}_{\delta}\left(\varepsilon_{\delta \alpha \beta} \hat{r}_{\gamma}+\varepsilon_{\delta \alpha \gamma} \hat{r}_{\beta}\right) \\
\boldsymbol{M}_{H}^{r d}(\boldsymbol{r}, a, b)=\sum_{\boldsymbol{n}}\left[\boldsymbol{M}_{O}^{r d}\left(\boldsymbol{r}_{\boldsymbol{n}}, a, b\right) \operatorname{erfc}\left(\frac{r_{\boldsymbol{n}}}{\sqrt{2 \sigma^{2}}}\right)+\left(\frac{1}{8 \sigma^{2}}+\frac{3}{8 r_{\boldsymbol{n}}^{2}}\right) \boldsymbol{q}\left(\hat{\boldsymbol{r}}_{\boldsymbol{n}}\right) \frac{1}{\sqrt{2 \pi^{3}} \eta \sigma} \mathrm{e}^{-r_{\boldsymbol{n}}^{2} / 2 \sigma^{2}}\right] \\
-\frac{1}{4 \eta V} \sum_{\boldsymbol{n}}^{\prime} \boldsymbol{q}\left(\hat{\boldsymbol{k}}_{\boldsymbol{n}}\right) \mathrm{e}^{-k_{\boldsymbol{n}}^{2} \sigma^{2} / 2} \cos \left(\boldsymbol{k}_{\boldsymbol{n}} \cdot \boldsymbol{r}\right)
\end{array}
$$

$$
\boldsymbol{M}_{O}^{d d}(\boldsymbol{r} ; a, b)=\frac{3}{40 \pi \eta a^{3} b^{3}}\left[f_{O}^{0}(r ; a, b) \boldsymbol{d}^{0}(\hat{\boldsymbol{r}})+f_{O}^{1}(r ; a, b) \boldsymbol{d}^{1}(\hat{\boldsymbol{r}})+f_{O}^{2}(r ; a, b) \boldsymbol{d}^{2}(\hat{\boldsymbol{r}})\right]
$$

$$
\begin{aligned}
& d_{\alpha \beta \gamma \delta}^{0}(\hat{\boldsymbol{r}})=\frac{3}{2}\left(\hat{r}_{\alpha} \hat{r}_{\beta}-\frac{1}{3} \delta_{\alpha \beta}\right)\left(\hat{r}_{\gamma} \hat{r}_{\delta}-\frac{1}{3} \delta_{\gamma \delta}\right), \quad d_{\alpha \beta \gamma \delta}^{1}(\hat{\boldsymbol{r}})=\frac{1}{2}\left(\hat{r}_{\alpha} \hat{r}_{\gamma} \delta_{\beta \delta}+\hat{r}_{\beta} \hat{r}_{\gamma} \delta_{\alpha \delta}+\hat{r}_{\alpha} \hat{r}_{\delta} \delta_{\beta \gamma}+\hat{r}_{\beta} \hat{r}_{\delta} \delta_{\alpha \gamma}-4 \hat{r}_{\alpha} \hat{r}_{\beta} \hat{r}_{\gamma} \hat{r}_{\delta}\right) \\
& d^{2}=\mathcal{I}-d^{0}-d^{1}
\end{aligned}
$$

$$
\begin{gathered}
f_{O}^{1}(r ; a, b)=a^{3} b^{3}\left(\frac{5}{r^{3}}-\frac{8\left(a^{2}+b^{2}\right)}{r^{5}}\right), \quad f_{O}^{2}(r ; a, b)=2 a^{3} b^{3} \frac{a^{2}+b^{2}}{r^{5}}, \\
f_{O}^{0}(r ; a, b)=-2 f_{O}^{1}(r ; a, b)-2 f_{O}^{2}(r ; a, b)
\end{gathered}
$$

The matrix $\boldsymbol{m}_{O, o v}^{d d}(\boldsymbol{r}, a, b)$ is given by a formula analogous to (A23) but with $f^{i}(r ; a, b)$ replaced by

$$
\begin{aligned}
& f_{O, o v}^{0}(r ; a, b)=\frac{1}{32 r^{5}}\left[5 r^{8}-30 r^{6}\left(a^{2}+b^{2}\right)+32 r^{5}\left(a^{3}+b^{3}\right)-10 r^{2}(a-b)^{4}\left(a^{2}+4 a b+b^{2}\right)+3(a-b)^{6}\left(a^{2}+6 a b+b^{2}\right)\right] \\
& f_{O, o v}^{1}(r ; a, b)=\frac{1}{32 r^{5}}\left[5 r^{8}-25 r^{6}\left(a^{2}+b^{2}\right)+32 r^{5}\left(a^{3}+b^{3}\right)-15 r^{4}\left(a^{2}-b^{2}\right)^{2}+5 r^{2}(a-b)^{4}\left(a^{2}+4 a b+b^{2}\right)-2(a-b)^{6}\left(a^{2}+6 a b+b^{2}\right)\right] \\
& f_{O, o v}^{2}(r ; a, b)=\frac{1}{64 r^{5}}\left[5 r^{8}-40 r^{6}\left(a^{2}+b^{2}\right)+64 r^{5}\left(a^{3}+b^{3}\right)-30 r^{4}\left(a^{2}-b^{2}\right)^{2}+(a-b)^{6}\left(a^{2}+6 a b+b^{2}\right)\right]
\end{aligned}
$$

$$
\begin{aligned}
\boldsymbol{M}_{H}^{d d}(\boldsymbol{r} ; a, b)=\sum_{\boldsymbol{n}}\left\{\boldsymbol{M}_{O}^{d d}\left(\boldsymbol{r}_{\boldsymbol{n}} ; a, b\right) \operatorname{erfc}\left(\frac{r_{\boldsymbol{n}}}{\sqrt{2} \sigma}\right)+\left(\phi^{0}\left(r_{\boldsymbol{n}} ; a, b\right) \boldsymbol{d}^{0}\left(\hat{\boldsymbol{r}}_{n}\right)+\boldsymbol{\phi}^{1}\left(r_{\boldsymbol{n}} ; a, b\right) \boldsymbol{d}^{1}\left(\hat{\boldsymbol{r}}_{n}\right)+\boldsymbol{\phi}^{2}\left(r_{\boldsymbol{n}} ; a, b\right) \boldsymbol{d}^{2}\left(\hat{\boldsymbol{r}}_{n}\right)\right)\right. \\
\quad+\frac{1}{20 \eta V} \sum_{\boldsymbol{n}}^{\prime}\left(-\frac{2}{3} k_{\boldsymbol{n}}^{2} \sigma^{2} \boldsymbol{d}^{0}\left(\hat{\boldsymbol{k}}_{\boldsymbol{n}}\right)+\boldsymbol{d}^{1}\left(\hat{\boldsymbol{k}}_{\boldsymbol{n}}\right)\right)\left(10-k_{\boldsymbol{n}}^{2}\left(a^{2}+b^{2}\right)\right) \mathrm{e}^{-\frac{1}{2} k_{n}^{2} \sigma^{2} \cos \left(\boldsymbol{k}_{\boldsymbol{n}} \cdot \boldsymbol{r}\right)} \\
\phi^{0}(r ; a, b)=\left(a^{2}+b^{2}\right)\left(-\frac{1}{15} \sigma^{-8} r^{4}+\frac{4}{15} \sigma^{-6} r^{2}+\frac{2}{5} \sigma^{-4}+\frac{12}{5} \sigma^{-2} r^{-2}+\frac{36}{5} r^{-4}\right)-\frac{2}{3} \sigma^{-4} r^{2}-2 \sigma^{-2}-6 r^{-2} \\
\phi^{1}(r ; a, b)=\frac{1}{10}\left(a^{2}+b^{2}\right)\left(r^{2} \sigma^{-6}-4 \sigma^{-4}-16 r^{-2} \sigma^{-2}-48 r^{-4}\right)+3 r^{-2}+\sigma^{-2} \\
\phi^{2}(r ; a, b)=\frac{2}{5}\left(a^{2}+b^{2}\right)\left(r^{-2} \sigma^{-2}+3 r^{-4}\right)
\end{aligned}
$$




$$
\widetilde{\boldsymbol{M}}_{H O}^{d d}(a)=-\frac{a^{2}}{25 \sqrt{2 \pi^{3}} \eta \sigma^{5}} \mathcal{I}
$$

${ }^{1}$ D. L. Ermak and J. A. McCammon, "Brownian dynamics with hydrodynamic interactions," J. Chem. Phys. 69, 1352 (1978).

${ }^{2}$ G. C. Ansell, E. Dickinson, and M. Ludvigson, "Brownian dynamics of colloidal-aggregate rotation and dissociation in shear flow," J. Chem. Soc. Faraday Trans. 2 81, 1269-1284 (1985).

${ }^{3}$ M. C. Bujan-Nunez and E. Dickinson, "Brownian dynamics simulation of a multi-subunit deformable particle in simple shear flow," J. Chem. Soc., Faraday Trans. 90, 2737-2742 (1994).

${ }^{4}$ A. Furukawa and H. Tanaka, "Key role of hydrodynamic interactions in colloidal gelation," Phys. Rev. Lett. 104, 245702 (2010).

${ }^{5}$ R. D. Groot, T. J. Madden, and D. J. Tildesley, "On the role of hydrodynamic interactions in block copolymer microphase separation," J. Chem. Phys. 110, 9739-9749 (1999).

${ }^{6} \mathrm{M}$. Ramia, D. Tullock, and N. Phan-Thien, "The role of hydrodynamic interaction in the locomotion of microorganisms," Biophys. J. 65, 755-778 (1993).

${ }^{7}$ G. Nägele, "On the dynamics and structure of charge-stabilized suspensions," Phys. Rep. 272, 215-372 (1996).

${ }^{8} \mathrm{P}$. Szymczak and M. Cieplak, "Influence of hydrodynamic interactions on mechanical unfolding of proteins," J. Phys.: Condens. Matter 19, 285224 (2007).

${ }^{9}$ S. D. Ryan, "Role of hydrodynamic interactions in chemotaxis of bacterial populations," Phys. Biol. 17, 016003 (2019).

${ }^{10} \mathrm{~T}$. Ando and J. Skolnick, "On the importance of hydrodynamic interactions in lipid membrane formation,” Biophys. J. 104, 96-105 (2013).

${ }^{11}$ T. Frembgen-Kesner and A. H. Elcock, "Striking effects of hydrodynamic interactions on the simulated diffusion and folding of proteins," J. Chem. Theory Comput. 5, 242-256 (2009).

${ }^{12} \mathrm{H}$. Tanaka, "Interplay between wetting and phase separation in binary fluid mixtures: roles of hydrodynamics," J. Phys.: Condens. Matt. 13, 4637 4674 (2001).

${ }^{13}$ J. Rotne and S. Prager, "Variational treatment of hydrodynamic interaction in polymers," J. Chem. Phys. 50, 4831-4837 (1969).

${ }^{14} \mathrm{H}$. Yamakawa, "Transport properties of polymer chains in dilute solution: Hydrodynamic interaction.” J. Chem. Phys. 53, 436-443 (1970).

${ }^{15} \mathrm{O}$. Byron, "Construction of hydrodynamic bead models from highresolution X-ray crystallographic or nuclear magnetic resonance data," Biophys. J. 72, 408-415 (1997).

${ }^{16} \mathrm{G}$. J. de la Torre, M. L. Huertas, and B. Carrasco, "Calculation of hydrodynamic properties of globular proteins from their atomic-level structure," Biophys. J. 78, 719-730 (2000)

${ }^{17} \mathrm{P}$. Zipper and H. Durchschlag, "Calculation of hydrodynamic parameters of proteins from crystallographic data using multibody approaches," Prog. Coll. Pol. Sci. 107, 58-71 (1997).

${ }^{18}$ P. J. Zuk, E. Wajnryb, K. A. Mizerski, and P. Szymczak, "Rotne-PragerYamakawa approximation for different-sized particles in application to macromolecular bead models," J. Fluid Mech. 741, R5 (2014).

${ }^{19}$ P. J. Zuk, B. Cichocki, and P. Szymczak, "GRPY: an accurate bead method for calculation of hydrodynamic properties of rigid biomacromolecules," Biophys. J. 115, 782-800 (2018).

${ }^{20}$ E. Wajnryb, K. A. Mizerski, P. J. Zuk, and P. Szymczak, "Generalization of the Rotne-Prager-Yamakawa mobility and shear disturbance tensors," J. Fluid Mech. 731, R3 (2013).

${ }^{21}$ K. A. Mizerski, E. Wajnryb, P. J. Zuk, and P. Szymczak, "The RotnePrager-Yamakawa approximation for periodic systems in a shear flow," $\mathrm{J}$ Chem. Phys. 140, 184103 (2014).

${ }^{22}$ P. J. Zuk, B. Cichocki, and P. Szymczak, "Intrinsic viscosity of macromolecules within the generalized Rotne-Prager-Yamakawa approximation," J. Fluid Mech. 822 (2017).

${ }^{23}$ L. Durlofsky, J. F. Brady, and G. Bossis, "Dynamic simulation of hydrodynamically interacting particles," J. Fluid Mech. 180, 21-49 (1987).

${ }^{24}$ J. F. Brady and G. Bossis, "Stokesian dynamics," Ann. Rev. Fluid Mech. 20, 111-157 (1988)
${ }^{25}$ E. Wajnryb, P. Szymczak, and B. Cichocki, "Brownian dynamics: divergence of mobility tensor," Physica A 335, 339-358 (2004).

${ }^{26}$ A. Lees and S. Edwards, "The computer study of transport processes under extreme conditions,' Journal of Physics C: Solid State Physics 5, 1921 (1972).

${ }^{27}$ D. J. Evans and G. Morriss, Statistical mechanics of nonequilibrium liquids (Cambridge University Press, 2008)

${ }^{28}$ A. Kraynik and D. Reinelt, "Extensional motions of spatially periodic lattices," Int. J. Multiphas. Flow 18, 1045-1059 (1992).

${ }^{29} \mathrm{H}$. Hasimoto, "On the periodic fundamental solutions of the stokes equations and their application to viscous flow past a cubic array of spheres," J. Fluid Mech 5, 317-328 (1959).

${ }^{30} \mathrm{C}$. W. J. Beenakker, "Ewald sum of the rotne-prager tensor," J. Chem. Phys. 85, 1581-1582 (1986)

${ }^{31}$ E. R. Smith, I. K. Snook, and W. van Megen, "Hydrodynamic interactions in Brownian dynamics. I. Periodic boundary conditions for computer simulations," Physica 143A, 441-467 (1987).

${ }^{32}$ J. F. Brady, R. J. Phillips, J. C. Lester, and G. Bossis, "Dynamic simulation of hydrodynamically interacting suspensions," J. Fluid Mech. 195, 257-280 (1988).

${ }^{33}$ B. Cichocki and B. Felderhof, "Periodic fundamental solution of the linear navier-stokes equations," Physica A 159, 19-27 (1989).

${ }^{34} \mathrm{H}$. Lorentz, "A general theory concerning the motion of a viscous fluid," Abhandl. Theor. Phys 1, 23 (1907).

${ }^{35} \mathrm{G}$. Perkins and R. Jones, "Hydrodynamic interaction of a spherical particle with a planar boundary i. free surface," Physica A: Statistical Mechanics and its Applications 171, 575-604 (1991).

${ }^{36} \mathrm{H}$. A. Lorentz, "A general theorem concerning the motion of a viscous fluid and a few consequences derived from it," Versl. Konigl. Akad. Wetensch. Amst. 5, 168-175 (1896).

${ }^{37}$ N. Liron and R. Shahar, "Stokes flow due to a Stokeslet in a pipe," J. Fluid. Mech. 86, 727-744 (1978).

${ }^{38} \mathrm{C}$. W. Oseen, Neuere methoden und ergebnisse in der Hydrodynamik (Akademische Verlagsgesellschaft, 1927).

${ }^{39} \mathrm{~S}$. Bhattacharya, J. Bławzdziewicz, and E. Wajnryb, "Hydrodynamic interactions of spherical particles in suspensions confined between two planar walls," J. Fluid Mech. 541, 263-292 (2005).

${ }^{40}$ A. Daddi-Moussa-Ider, M. Lisicki, S. Gekle, A. M. Menzel, and H. Löwen, "Hydrodynamic coupling and rotational mobilities near planar elastic membranes," J. Chem. Phys. 149, 014901 (2018).

${ }^{41} \mathrm{~B}$. Felderhof, "Mobility of a particle immersed in a liquid film between two fluids," J. Chem. Phys. 124, 124705 (2006)

${ }^{42}$ Y. Sokolov and H. Diamant, "Many-particle mobility and diffusion tensors for objects in viscous sheets," J. Chem. Phys. 149, 034901 (2018).

${ }^{43}$ C. Stoltz, J. J. de Pablo, and M. D. Graham, "Concentration dependence of shear and extensional rheology of polymer solutions: Brownian dynamics simulations," J. Rheol. 50, 137-167 (2006)

${ }^{44}$ A. Jain, P. Sunthar, B. Dünweg, and J. R. Prakash, "Optimisation of a brownian-dynamics algorithm for semidilute polymer solutions," Phys. Rev. E 85, 066703 (2012)

${ }^{45}$ T. Zhou and S. B. Chen, "Computer simulations of diffusion and dynamics of short-chain polyelectrolytes," J. Chem. Phys. 124, 034904 (2006).

${ }^{46}$ A. M. Fiore, F. Balboa Usabiaga, A. Donev, and J. W. Swan, "Rapid sampling of stochastic displacements in brownian dynamics simulations," J. Chem. Phys. 146, 124116 (2017).

${ }^{47}$ A. M. Fiore and J. W. Swan, "Rapid sampling of stochastic displacements in brownian dynamics simulations with stresslet constraints," J. Chem. Phys. 148, 044114 (2018).

${ }^{48}$ A. M. Fiore and J. W. Swan, "Fast stokesian dynamics," J. Fluid Mech. 878 544-597 (2019).

${ }^{49} \mathrm{~B}$. Cichocki and R. Jones, "Image representation of a spherical particle near a hard wall," Physica A 258, 273-302 (1998).

${ }^{50} \mathrm{G}$. Bossis and J. F. Brady, "Dynamic simulation of sheared suspensions. I. 
General method,” J. Chem. Phys. 80, 5141-5154 (1984).

${ }^{51}$ A. Sierou and J. F. Brady, "Shear-induced self-diffusion in non-colloidal suspensions," J. Fluid. Mech. 506, 285-314 (2004).

${ }^{52}$ D. I. Dratler, W. R. Schowalter, and R. L. Hoffman, "Dynamic simulation of shear thickening in concentrated colloidal suspensions," J. Fluid. Mech. 353, 1-30 (1997).

${ }^{53}$ R. C. Ball and J. R. Melrose, "A simulation technique for many spheres in quasi-static motion under frame-invariant pair drag and Brownian forces," Physica A 247, 444-472 (1997).

${ }^{54}$ S. L. Dance, E. Climent, and M. R. Maxey, "Collision barrier effects on the bulk flow in a random suspension," Phys. Fluids 16, 828-831 (2004).

${ }^{55} \mathrm{G}$. Bossis and J. F. Brady, "Dynamic simulation of sheared suspensions. I. General method,” J. Chem. Phys. 80, 5141-5154 (1984).
${ }^{56}$ D. I. Dratler and W. R. Schowalter, "Dynamic simulation of suspensions of non-Brownian hard spheres," J. Fluid. Mech. 325, 53-77 (1996).

${ }^{57}$ M. L. Ekiel-Jeżewska, T. Gubiec, and P. Szymczak, "Stokesian dynamics of close particles," Phys. Fluids 20, 063102 (2008).

${ }^{58}$ A. J. C. Ladd, "Dynamical simulations of sedimenting spheres," Phys. Fluids 5, 299-310 (1993)

${ }^{59}$ J. W. Swan and J. F. Brady, "Simulation of hydrodynamically interacting particles near a no-slip boundary," Phys. Fluids 19, 113306 (2007).

${ }^{60} \mathrm{~F}$. Balboa Usabiaga, B. Delmotte, and A. Donev, "Brownian dynamics of confined suspensions of active microrollers," J. Chem. Phys. 146, 134104 (2017). 


\section{(a)}

$\boldsymbol{v}_{\infty}(\boldsymbol{r})=(\dot{\gamma} z, 0,0)$

$\dot{\gamma} L_{z} t \bmod L_{x}$

$\vdots$
$\vdots$
$\vdots$
$\vdots$
$\vdots$
$\vdots$

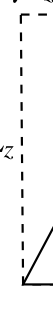

$$
\boldsymbol{n}=[0,0,1] \quad n=[1,0,1]
$$

(b)

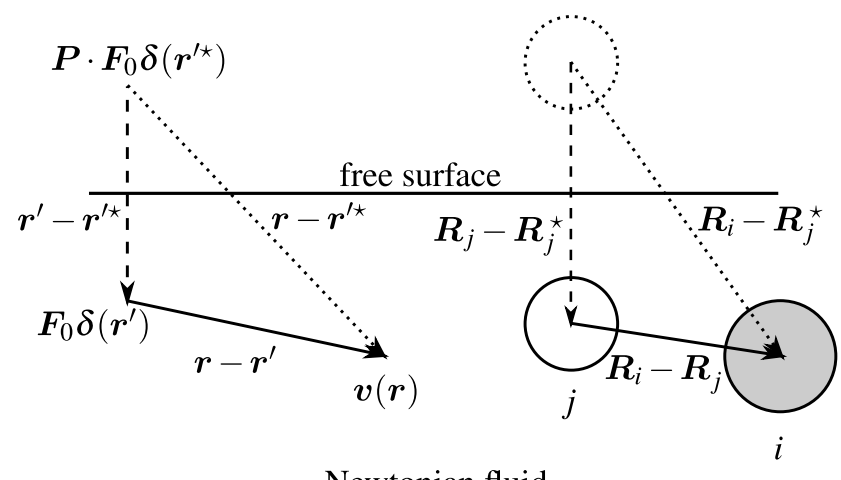

Newtonian fluid 